\title{
Notes to the Reader
}

The Chinese language is complex, with many different dialects that vary widely in their pronunciation. Throughout the text, the standard Mandarin (pinyin) is generally used. When pinyin is not used, the spelling of Chinese terms and names reflects the version given by the sources. The Hakka language is used with Hakka classic dishes and the names of some places in the Hakka heartland.

In the Hakka Pantry (page 247), standard Mandarin and several common dialects in phonetic spellings are included to provide the most help in finding ingredients. The phonetic spelling of Chinese words varies depending on the dialect used. The Chinese characters in that section are written in the traditional way.

Chinese people traditionally place their family name first, followed by their first name (usually two words, sometimes hyphenated or written together as one word). However, many Chinese, especially those who live in the West, reverse the order and state their given names followed by their surnames. Or they may adopt a Western first name. I have stated their names as they prefer or as commonly used.

If you're new to Chinese cooking, please read through the Hakka Kitchen (page 235) first to acquaint yourself with the cuisine's basic techniques and equipment. If you run across unfamiliar ingredients, check the Hakka Pantry. Before you begin a recipe, read through the instructions and prepare all ingredients for cooking; this is especially important for fast-paced stir-fries. Don't be put off by long recipes. The detailed steps will guide you through unfamiliar ingredients and techniques to successful and delicious results. 